International Journal of Biomedicine I June 2019 - Volume 9, Issue Suppl_1: Abstracts From the Second Russian International Conference "Cryo-electron microscopy 2019: achievements and prospects"

POSTER ABSTRACT PRESENTATIONS

SESSION TITLE: MOLECULAR ORGANIZATION OF CELLS AND ORGANELLES

DOI: 10.21103/IJBM.9.Suppl_1.P25

\title{
Abstract P-25: Cryo-Electron Microscopy of Extracellular Vesicles from Cerebrospinal Fluid
}

Tatiana A. Shtam ${ }^{1}$, Anton K. Emelyanov ${ }^{1,2}$, Roman A. Kamyshinsky ${ }^{1,5}$, Luiza A. Garaeva ${ }^{1,2}$, Nikolay A. Verlov ${ }^{1}$, Anastasia Kudrevatykh ${ }^{3}$, Gaspar Gavrilov ${ }^{4}$, Sofia N. Pchelina ${ }^{1,2,3}$

${ }^{1}$ Petersburg Nuclear Physics Institute named by B.P. Konstantinov of NRC «Kurchatov Institute», St. Petersburg, Russia; ${ }^{2}$ Pavlov First Saint Petersburg State Medical University, St. Petersburg, Russia; ${ }^{3}$ Institute of Experimental Medicine, St. Petersburg, Russia; ${ }^{4}$ S.M. Kirov Saint-Petersburg Military Medical Academy, St. Petersburg, Russia.; "National Research Center "Kurchatov Institute”, Moscow, Russia

Background: Extracellular vesicles (EVs) are membrane-enclosed vesicles which play important role for cell communication. EVs are found in many human biological fluids; contain proteins, nucleic acids and lipids. These vesicles deliver biological information to recipient cells thereby modulating their behaviors. In this way, EVs are involved in the pathological development of many human disorders, including neurodegenerative diseases. Definition of the EV size and morphology is important for studying of their participation in the intercellular signaling pathways in pathology and normal state. Especially for understanding the role of EVs in the pathogenesis of brain, detailed characterization of the vesicles from cerebrospinal fluid seems to be the most relevant.

Methods: In this study, EVs purified from cerebrospinal fluid or plasma of patients with Parkinson's disease were characterized using cryo-electron microscopy (cryo-EM), nanoparticle tracking analysis (NTA) and flow cytometry.

Results: The size of the observed vesicles and the presence of protein exosomal marker on the membrane confirmed by NTA and flow cytometry, suggest that most of the vesicles were represented as exosomes. According to the NTA analysis the concentration EVs in the cerebrospinal fluid $((6.1 \pm 4.9)$ $\times 10^{9}$ particles $\left./ \mathrm{ml}\right)$ is two orders of magnitude lower than that in blood plasma $\left((19.8 \pm 14.9) \times 10^{9}\right.$ particles/ml). Vesicles isolated from cerebrospinal fluid were examined in detail using cryo-EM. EVs of various sizes and morphology with lipid bilayer and vesicle internal structures were observed. More than 80 percent of the particles were classified as vesicles due to the clear presence of lipid bilayer membrane. The majority of vesicles was intact and had a round shape. Single (74.9 $\pm 27.24 \mathrm{~nm})$, double $(179.49 \pm 105.29 \mathrm{~nm})$ and multilayer vesicles $(206.18 \pm 78.3)$ were visualized. We found that multilayer 
vesicles were larger than the single vesicle $(\mathrm{P}<0.0001)$. Vesicles were assigned into multilayer category when two or more vesicles were contained inside a larger one. Various combinations, having from two to six vesicles inside the one were also found.

Conclusion: Here, we described the characteristics of the vesicles from cerebrospinal fluid and found their variety suggesting that subpopulations of EVs with different and specific functions may exist.

Key Words: cryo-electron microscopy $\bullet$ cerebrospinal fluid $\bullet$ extracellular vesicles $\bullet$ Parkinson's disease

Sources of Funding: Cryo-EM experiments were supported by the RSF (project 19-74-20146). All other experiments were supported by the RSF (project 17-75-20159).

International Journal of Biomedicine. 2019;9 Suppl 1: S27-28. doi: 10.21103/JJBM.9.Suppl_1.P25

(C)2019 International Medical Research and Development Corporation 